\title{
Giro dinámico y lógica de la investigación científica ${ }^{\dagger}$
}

\section{Dynamic turn and logic of scientific research}

\author{
Ma Victoria Murillo-Corchado ; Ángel Nepomuceno-Fernández \\ *Universidad de Sevilla, España \\ mvmurilloc@gmail.com \\ **Universidad de Sevilla, España \\ nepomuce@us.es
}

DOI: https://doi.org/10.22370/rhv2019iss13pp68-89

Recibido: 25/07/2019. Aceptado: 16/08/2019

\section{Resumen}

Para presentar la incidencia del giro dinámico en la lógica de la investigación científica, en este artículo comenzamos con una sección que trata de los juegos lógicos como desencadenantes de este giro dinámico en la lógica contemporánea, junto con el programa de dinámica lógica de la información y la interacción. Sucintamente presentamos las principales características de la lógica favorable a la independencia y la semántica juego-teórica (IF-logic y GTS, respectivamente, en Hintikka y Sandu 1997), de la lógica dialógica (Redmond y Fontaine 2011), así como los elementos esenciales de dicho programa. Si bien a partir de cualquiera de estos puntos de vista se cuenta con un elenco de herramientas lógicas para abordar cuestiones más claramente epistemológicas, destacamos el papel de la lógica epistémica dinámica (LED), a la que dedicamos la siguiente sección. Sigue otra en la que entramos en los estudios lógicos de la abducción como uno de los problemas fundamentales de la epistemología contemporánea y, en una nueva sección, en términos de los constructos teóricos de las secciones precedentes, presentamos y explicamos un fenómeno surgido en el campo de la lingüística, el caso del descubrimiento de la lengua amazónica pirahã, que debería ser considerado una anomalía en el marco de la teoría chomskiana.

‘Este trabajo se ha realizado en el marco de las actividades que lleva a cabo el Grupo de Investigación en Lógica, Lenguaje e Información de la Universidad de Sevilla (GILLIUS http://grupo.us.es/ghum609/php/ node/41), referencia HUM-609, del Plan Andaluz de Investigación, Desarrollo e Innovación. 
Palabras clave: juegos lógicos, lógica dialógica, LED, abducción, gramática universal, pirahã, anomalía, novedad.

\begin{abstract}
In order to present the incidence of the dynamic turn in the logic of scientific research, we begin with a section, in this article, that deals with logical games as triggers of this dynamic turn in contemporary logic, together with the program of logical dynamics of information and interaction. We briefly introduce the main characteristics of the logic favorable to independence and the game-theoretical semantics (IF-logic and GTS, respectively, in Hintikka and Sandu 1997), of dialogical logic (Redmond and Fontaine 2011), as well as the essential elements of this program. Although from any of these points of view we have a list of logical tools to deal with more clearly epistemological issues, we highlight the role of dynamic epistemic logic (DEL), to which we dedicate the following section. There follows another one in which we enter the logical studies of abduction as one of the fundamental problems of contemporary epistemology and, in a new section, in terms of the theoretical constructs of the preceding sections, we present and explain a phenomenon that emerged in the field of linguistics, the case of the discovery of the Amazonian language pirahã, which should be considered an anomaly within the framework of the Chomskian theory.
\end{abstract}

Keywords: Logical games, dialogical logic, DEL, abduction, universal grammar, pirahã, anomaly, novelty.

\title{
1. Introducción
}

El giro dinámico en lógica, por cuanto éste representa en términos kuhnianos un cambio de paradigma en la investigación lógica actual, tiene lugar a partir de la aparición de la semántica juego-teórica (GTS) según señala P. Gochet: "The invention of game-theoretic semantics by J. Hintikka in the seventies (Hintikka 1973; Saarinen 1979) can be described as the emergence of a new paradigm not only for semantics but also for logic and the philosophy of mathematics" (Gochet 2002, 175). Aceptando esta observación, añadimos que el giro en cuestión tiene como ingredientes, de un lado, las propuestas de estudiar las tareas lógicas como si éstas fueran desarrollos de formas de juego y, de otro, el vasto programa denominado "dinámica lógica de la información y de la interacción", propuesto en van Benthem (2011).

Por otra parte, las bases para una lógica de la investigación científica ya no se establecen, bajo el supuesto de que ello es posible, exclusivamente en términos de lógica clásica. Más bien son las lógicas no clásicas las que proporcionan las herramientas necesarias para el abordaje de este tipo de problemas epistemológicos. La noción de abducción debida a Peirce, a pesar de su carácter poliédrico, permite avanzar en las relaciones entre 
lógica y epistemología, de manera que, si hacemos la tradicional distinción entre contexto de descubrimiento y contexto de justificación, el primero no es ajeno a la lógica misma, en contra de la opinión de autores como Popper, según el cual el contexto de descubrimiento no requería un análisis lógico por ser temática más bien de la psicología experimental (Popper 1980: 30-31). Ahora bien, "en la epistemología de Peirce, el pensamiento es un proceso dinámico, esencialmente una acción que oscila entre los estados mentales de duda y de creencia" (Aliseda 2014, 46).

\section{Juegos lógicos}

Al producirse el giro dinámico, surgen varias propuestas para entender las tareas lógicas en forma de juegos, con lo que las nociones lógicas básicas emergen uniformemente como estrategias ganadoras en diversos escenarios. Las principales propuestas son:

1. Hintikka, GTS. Se trata de hacer una evaluación de juegos entre un Verificador y un Falsificador, teniendo en cuenta un oráculo (un modelo).

2. Ehrenfeucht-Fraïssé. Se trata de juegos entre un Duplicador y un Estropeador - spoiler-que comparan modelos.

3. Lorenz-Lorenzen. Establecen juegos de diálogos argumentativos entre un Proponente y un Oponente.

Uno de los ingredientes del caldo de cultivo que propicia la aparición del nuevo paradigma es el origen mismo de los computadores modernos y el diseño de máquinas de deducción lógica. Surge así un extraordinario interés por los fenómenos de la comunicación en general, un mundo cognitivo en el que se puede situar el dominio de la lógica, como "el estudio de los invariantes subyacentes en estos procesos informacionales" (van Benthem 2011, 1). Así pues, son una gama de procesos informacionales los que deben constituir la teoría lógica. Ahora bien, para trabajar en este ámbito se plantea la necesidad de abordar la interacción entre estructuras estáticas y dinámicas.

Se puede decir que tanto la lógica clásica como los avances en la tradición algebraica convergen finalmente en los planteamientos de los juegos lógicos y la dinámica lógica de la información, aunque se ha señalado a Peirce como un precedente inmediato. En efecto, para el lógico norteamericano el hablante es esencialmente un defensor de su propia proposición y su deseo es interpretarla de manera que sea defendible, mientras que el intérprete no está tan interesado en interpretarla plenamente sin considerar a qué extremo se puede llegar, es relativamente hostil y busca la interpretación menos defendible.

Considerada la actividad lógica como un juego — en este punto Hintikka se considera un seguidor de Wittgenstein, posición discutible para algunos (Marion 2006)—, se había de replantear el sentido de las reglas de inferencia. Para justificar una inferencia, Hintikka propone dos clases de reglas o principios, a saber, las reglas definitorias, similares a las que definen un juego, como, por ejemplo, en el ajedrez - la deducción o la indagación 
científica contienen los elementos de un juego estratégico-. Estas reglas nos indican los movimientos posibles en una situación dada (en el curso de un juego como el ajedrez, este tipo de reglas nos dicen cómo se mueven la reina, el alfil, la torre, etc.). Hay otras reglas, las estratégicas, que nos indican cuáles son los mejores movimientos para ganar el juego (o evitar que nos gane el contrincante; en el caso del ajedrez, cómo evitar un jaque pastor, etc.). Además, Hintikka establece una lógica de cuestiones y respuestas, que tiene en cuenta las diferencias entre razonamiento ampliativo y no ampliativo - distinción que se da entre pasos de un argumento: interrogativo (ampliativos) y deductivos (no ampliativos) - En la indagación interrogativa la cuestión es anticipar la situación epistémica provocada por la respuesta. Todas estas observaciones deberían tenerse en cuenta como un importante conjunto de consejos certeros para abordar aproximaciones lógicas a la inferencia científica.

Desde la GTS propuesta para interpretar la lógica favorable a la independencia (If-logic, Hintikka 1997; Hintikka y Sandu 1997; Aho y Pietarinen 2006), donde el lenguaje se considera orientado a metas más que como una actividad gobernada por reglas, conocer el significado de una oración es conocer el cambio en el estado de información, que parece ampliar. A partir de la idea de actualización de la información, cabe definir una noción de actualización mínima (van Benthem 2011), siempre que los estados cognitivos (o estados de información) sean ordenados por inclusión. A diferencia de la semántica tarskiana, que adopta el principio de composicionalidad —el significado de una proposición compleja viene dado por el significado de las proposiciones más simples que la integran-, el punto de vista de la GTS toma como base el principio de significado dependiente del contexto y considera que éste resulta incompatible con el principio de composicionalidad - el significado de una proposición compleja es una función del significado de sus partes constituyentes-, incompatibilidad que podría ser eliminada cambiando la concepción estática del significado por otra de carácter dinámico.

Dados un modelo $\mathrm{M}$ y una fórmula $\varphi$ (para simplificar, nos mantendremos a nivel proposicional), un juego semántico asociado al modelo $\mathrm{M}$ y la fórmula $\varphi$, en símbolos $\mathrm{G}$ ( $\mathrm{M}$, $\varphi)$, caracteriza la verdad o falsedad de la fórmula en el juego semántico, que es jugado por dos jugadores, Abelardo y Eloísa, que se pueden representar como A y E, respectivamente. En el juego G $(M, \varphi)$ tanto A como E han de hacer una elección, de acuerdo con unas reglas establecidas para cada clase de fórmulas, es decir por las reglas constitutivas de $\mathrm{G}(\mathrm{M}, \varphi)$. Estas son:

1. $\varphi$ es atómica. No se hace ningún movimiento:

a. Si $\mathrm{M} \vDash \varphi$, entonces $\mathrm{E}$ ha ganado,

b. Si $\mathrm{M} \vDash \varphi$, entonces A ha ganado.

2. $\varphi=\delta \vee \psi$ : E elige $\theta \in\{\delta, \psi\}$ y el juego continúa como $\mathrm{G}(\mathrm{M}, \theta)$.

3. $\varphi=\delta \wedge \psi$ : como antes, sólo que $\mathrm{A}$ es quien hace la elección. 
4. $\varphi=\neg \psi$ : lo mismo que en el juego $\mathrm{G}(\mathrm{M}, \psi)$, excepto que los papeles de los jugadores A y E cambian, incluyendo las reglas de ganancia y pérdida.

Asimismo, hemos de considerar las reglas de estrategia, teniendo en cuenta que cada juego $\mathrm{G}(\mathrm{M}, \varphi)$ es un juego de información perfecta, los jugadores "conocen" todas las elecciones hechas en el desarrollo del juego — se pueden considerar también de información imperfecta-. Al final del juego, uno de los jugadores gana y el otro pierde, de acuerdo con la circunstancia de que la fórmula atómica con la cual termina el juego sea verdadera o falsa en el modelo (que pertenezca o no a $\mathrm{M}$ ). Una estrategia de un jugador $\mathrm{X}$ en un juego $\mathrm{G}(\mathrm{M}, \varphi)$ no es más que un método que produce un movimiento (legal) para $\mathrm{X}$ contra cualquier secuencia de movimientos hecha por su oponente. Para una fórmula $\varphi$ y un modelo $M$, una estrategia para un jugador $X$ es un conjunto $F_{i}$ de funciones $f_{Q}$, correspondientes a las distintas constantes lógicas Q, que pueden incitar a un movimiento de $X$ en $G(M, \varphi)$. Es una estrategia ganadora para el jugador $X$, si lleva a la victoria de $X$ contra cualquier movimiento del oponente.

El estudio del valor de verdad se hace atendiendo a las siguientes estipulaciones, para un modelo $\mathrm{M}$ y una fórmula $\varphi$ :

1. $\varphi$ es verdadera (en el sentido de la semántica juego-teórica - GTS-) syss existe una estrategia ganadora para $\mathrm{E}$ en el juego $\mathrm{G}(\mathrm{M}, \varphi)$; simbólicamente:

$$
\mathrm{M} \vDash{ }_{\text {GTS }}^{+} \varphi
$$

2. $\varphi$ es falsa (en el sentido de la semántica GTS) syss existe una estrategia ganadora para A en $\mathrm{G}(\mathrm{M}, \varphi)$; simbólicamente:

$$
\mathrm{M} \models_{\text {GTS }}^{-} \varphi
$$

El otro punto de vista significativo es el de la elaboración de una lógica dialógica. Se puede decir que existe una relación entre los diálogos y las reglas de razonamiento correcto (dialéctica, obligaciones, etc.). A mediados del s. XX, P. Lorenzen puso en relación diálogos y fundamentos constructivos de la lógica. Se define un diálogo D sobre una proposición (en su caso, una fórmula) $\varphi-\mathrm{D}(\varphi)-$, que comienza con $\varphi$ afirmada por un jugador, y alcanza una posición final con victoria o derrota después de un número finito de movimientos de acuerdo con reglas definidas (Rahman, Clerbout y Keiff 2009; Redmond y Fontaine 2011). La noción dialógica de demostración descansa en esta noción de juego. En cuanto a la estructura de un juego dialógico, se considera que en el diálogo participan un proponente $(\mathrm{P})$ y un oponente $(\mathrm{O})$, que discuten una tesis siguiendo ciertas reglas, ejecutando los movimientos de ataque o de defensa. Las reglas se dividen en:

1. De partícula (de las constantes lógicas), que muestran qué movimientos están autorizados para atacar los movimientos del otro jugador o defender los propios.

2. Estructurales, que determinan el curso general del juego dialógico. Estas a su vez son: 
a. Inicial: la fórmula es afirmada por P. Alternativamente habrá movimientos de $\mathrm{P}$ y de $\mathrm{O}$. Cada movimiento que sigue al inicial es un ataque o una defensa.

b. Situación: P y O sólo pueden hacer movimientos que cambien la situación.

c. Formal: P no puede introducir fórmulas atómicas, salvo que previamente las haya afirmado $\mathrm{O}$.

d. Ganancia: X gana syss es el turno de Y pero éste no puede hacer ningún movimiento.

e. Intuicionista: en cada movimiento, cada jugador puede atacar una fórmula compleja afirmada por el otro o puede defenderse contra el último ataque que no ha sido aún defendido.

f. Clásica: en cualquier movimiento un jugador puede atacar una fórmula compleja afirmada por el otro o puede defenderse contra cualquier ataque (incluyendo aquellos que ya han sido defendidos).

En la construcción de diálogos se usará la regla intuicionista o la regla clásica, según la finalidad que se persiga. Este planteamiento no ha estado exento de críticas en las cuales se ha considerado la lógica dialógica como exótica y se ha afirmado que es una lógica constructiva. En su origen, en efecto, fue propuesta como lógica intuicionista, sin embargo los posteriores desarrollos la han convertido en una especie de marco general, a partir del cual se pueden establecer diálogos para otras lógicas (modal, epistémica, etc.) $\mathrm{y}$, aunque hace uso de alguna terminología propia, no complica demasiado las prácticas más que otros procedimientos (ya sean secuentes, tableaux, etc.). Este planteamiento ofrece una dimensión pragmática de las constantes lógicas. Desde luego constituyen una proto-semántica: esquema de juego que al completarse con reglas estructurales presenta la semántica del juego. Los operadores lógicos forman juegos desde otros juegos más simples, que muestran cómo relacionar sentencias y proposiciones; la aserción de una sentencia contiene una proposición con cierta fuerza conferida por el ataque (demanda de proferir una afirmación) y la defensa (respuesta de que se puede proferir la aserción).

En cualquier caso, se trata de una importante manifestación del giro dinámico que venimos comentando y se puede considerar afín a los planteamientos de la lógica favorable a la independencia y la correspondiente GTS.

Aunque el punto de vista de los juegos lógicos ha sido fundamental para la aparición del giro dinámico en lógica, hemos de destacar el desarrollo de la lógica epistémica dinámica propiciada en el mismo como elemento esencial (Baltag y Smets 2012), sobre todo los avances logrados en el marco de la dinámica lógica de la información (van Benthem 2011), en el que se ha planteado un amplio proyecto de investigación a cuya base está la consideración de la inferencia como un proceso informacional. Cabe decir, a este respecto, que las actividades de inferencia, evaluación, revisión de creencias, etc. 
son tan importantes como sus correspondientes productos en la configuración de la teoría lógica. Basta observar cualquier proceso informacional para comprobar que en realidad ha habido observación, comunicación e inferencia, incluso que se han barajado cuestiones y respuestas. La información inicial se va modificando con las distintas acciones. Un sencillo puzle lo ejemplifica. Sobre una reunión en la Universidad se sabe que, si asiste el decano o el vicerrector, entonces asiste el director del departamento; si no asiste el decano, entonces asiste el vicerrector; si asiste el vicerrector, no asiste el director. Usando las minúsculas iniciales correspondientes, se formalizan estas reglas como:

$$
\mathrm{d} \vee \mathrm{v} \rightarrow \mathrm{t} ; \neg \mathrm{d} \rightarrow \mathrm{v} ; \mathrm{v} \rightarrow \neg \mathrm{t} .
$$

El estado inicial de información lo constituyen las 8 opciones — cada literal positivo representa que la correspondiente circunstancia se da, pero si es negativo, que no se da-

$$
\{\mathrm{dvt}, \mathrm{dv} \neg \mathrm{t}, \mathrm{d} \neg \mathrm{vt}, \neg \mathrm{dvt}, \mathrm{d} \neg \mathrm{v} \neg \mathrm{t}, \neg \mathrm{dv} \neg \mathrm{t}, \neg \mathrm{d} \neg \mathrm{vt}, \neg \mathrm{d} \neg \mathrm{v} \neg \mathrm{t}\}
$$

Cada una de las afirmaciones indicadas da lugar a una actualización del estado inicial:

1. $\mathrm{d} \vee \mathrm{v} \rightarrow \mathrm{t}$. Nuevo estado $\{\mathrm{dvt}, \mathrm{d} \neg \mathrm{vt}, \neg \mathrm{dvt}, \neg \mathrm{d} \neg \mathrm{vt}, \neg \mathrm{d} \neg \mathrm{v} \neg \mathrm{t}$ \}

2. $\neg \mathrm{d} \rightarrow \mathrm{v}$. El nuevo estado (se eliminan más opciones) es $\{\mathrm{dvt}, \mathrm{d} \neg \mathrm{vt}, \neg \mathrm{dvt}\}$

3. $\mathrm{v} \rightarrow \neg \mathrm{t}$. Entonces el nuevo estado es $\{\mathrm{d} \neg \mathrm{vt}\}$

Así pues, el resultado es que a la reunión asiste el decano, no asiste el vicerrector y también asiste el director del departamento.

Otro ejemplo habitual es el de tres individuos, sean 1, 2 y 3 , integrantes de un jurado, que debe elegir entre A y B (finalistas de un concurso, o dos individuos que optan a cualquier otro tipo de premio). Cada uno apunta su voto en una nota. El secretario es distinto de 1, 2 y 3 y no vota, examina las notas y sólo dice "no hay consenso". Entonces 2 muestra a 1 su voto y declaran que ellos dos no han votado lo mismo. En ese momento, 1 y 2 desconocen el resultado. Sin embargo, 3 lo conoce perfectamente. En efecto, puesto que el voto de 3 coincide con uno de los otros dos - de acuerdo con la información hecha pública por el secretario y los otros dos miembros del jurado-, 3 ya sabe que ha ganado justo quien ha sido votado por él. Se suelen dar otros ejemplos, como el de los niños con la frente manchada, a partir de los cuales se introducen las cuestiones más relevantes que se presentan en el tratamiento lógico del conocimiento (véase Fagin et al. 1995).

En última instancia, el propio giro dinámico en lógica y semántica ha inspirado la aparición y el desarrollo de la lógica epistémica dinámica (LED), su columna vertebral, que se ha beneficiado de la confluencia de esfuerzos por parte de investigadores cuyo trabajo se realiza en diversas disciplinas, principalmente, filosofía, lingüística, álgebra, ciencias de la computación y teoría de juegos, entre otras, lo que le da un marcado sello de interdisciplinariedad. 


\section{Lógica epistémica dinámica}

La lógica epistémica es una extensión de la lógica clásica que estudia operadores epistémicos. En esta breve presentación de LED seguimos a van Ditmarsch, van der Hoek y Kooi (2008), con ligeras modificaciones de notación. Dado un conjunto de variables proposicionales $\mathcal{P} \neq \varnothing$, un lenguaje proposicional básico para LED se define de acuerdo con la siguiente regla $\mathrm{BNF}$,

$$
\varphi::=\mathrm{p}|\perp| \neg \varphi|\varphi \vee \mathcal{X}| \varphi \wedge \mathcal{X}|\varphi \rightarrow \mathcal{X}| \mathrm{K}_{\mathrm{a}} \varphi \mid[\varphi !] \mathcal{X}
$$

donde p es una variable proposicional; $\perp$ es una constante proposicional (contradicción); $\mathrm{K}_{\mathrm{a}} \varphi$ representa que el agente a conoce $\varphi ; \mathrm{a} \in \mathrm{G}$, el conjunto de todos los agentes; $[\varphi !] \chi$ indica que tras cada anuncio de $\varphi, \mathcal{X}$ es el caso. El operador de conocimiento $\mathrm{K}$, como los operadores modales en general, tiene su dual, a saber $\hat{\mathrm{K}}$, el cual, para el agente a, es definible como $\hat{\mathrm{K}}_{\mathrm{a}} \varphi=\neg \mathrm{K}_{\mathrm{a}} \neg \varphi$, que se lee hasta donde el agente a conoce, $\varphi$ es posible.

La semántica para este lenguaje proposicional se suele establecer en términos kripkeanos. Un modelo

$$
\mathrm{M}=\left\langle\mathrm{W},\left\{\Re_{\mathrm{a}}: \mathrm{a} \in \mathrm{G}\right\}, \mathrm{v}\right\rangle,
$$

Donde $\mathrm{W} \neq \varnothing$ es el conjunto de estados (o mundos); $\left\{\Re_{\mathrm{a}}: \mathrm{a} \in \mathrm{G}\right\}$ es un conjunto de relaciones definidas en $\mathrm{W}$ para cada individuo $\mathrm{a} \in \mathrm{G}$ (son las relaciones de accesibilidad) —escribiremos sólo $\Re_{\mathrm{a}}$, no $\left\{\Re_{\mathrm{a}}: \mathrm{a} \in \mathrm{G}\right\}$ - $\mathrm{v}: \mathcal{P} \rightarrow 2^{\mathrm{w}}$, asigna a cada variable proposicional el conjunto de estados (mundos) en los que vale la variable, de manera que $\mathrm{v}(\perp)=\varnothing$ $\mathrm{y} \mathrm{v}(\mathrm{p}) \in \wp(\mathrm{W})-\mathrm{o}$, lo que es lo mismo, $\mathrm{v}(\mathrm{p}) \subseteq \mathrm{W}-$.

A veces se menciona el conjunto de estados $\mathrm{W}$ como el dominio del modelo, por lo que se puede decir que $\mathrm{D}(\mathrm{M})=\mathrm{W}$, $\mathrm{y}$, puesto que $\mathrm{M}$ es un modelo epistémico, para referirnos al modelo epistémico en el estado w, se anotará M, w. Teniendo en cuenta estas consideraciones, a partir de un modelo epistémico $\mathrm{M}=\left\langle\mathrm{W}, \Re_{\mathrm{a}}, \mathrm{v}\right\rangle$ y el estado $\mathrm{s} \in \mathrm{W}$, el modelo en tal estado satisface una fórmula $\varphi$, simbólicamente, $\mathrm{M}, \mathrm{s} \vDash \varphi$, según el siguiente clausulado

- $\mathrm{M}, \mathrm{s} \vDash \mathrm{p}$ syss $\mathrm{s} \in \mathrm{v}(\mathrm{p})$,

- $\mathrm{M}, \mathrm{s} \vDash \neg \varphi$ syss $\mathrm{M}, \mathrm{s} \not \models \varphi$,

- $\mathrm{M}, \mathrm{s} \vDash \varphi \vee \psi$ syss $\mathrm{M}, \mathrm{s} \vDash \varphi$ o $\mathrm{M}, \mathrm{s} \vDash \psi$,

- $\mathrm{M}, \mathrm{s} \models \varphi \wedge \psi$ syss $\mathrm{M}, \mathrm{s} \vDash \varphi$ y $\mathrm{M}, \mathrm{s} \vDash \psi$,

- $\mathrm{M}, \mathrm{s} \vDash \varphi \rightarrow \psi$ syss $\mathrm{M}, \mathrm{s} \vDash \neg \varphi$ o $\mathrm{M}, \mathrm{s} \models \psi$,

- $\mathrm{M}, \mathrm{s} \vDash \mathrm{K}_{\mathrm{a}} \varphi$ syss para todo s' $\in \mathrm{W}$, si $\mathrm{R}_{\mathrm{a}}$ (s, s'), entonces $\mathrm{M}, \mathrm{s}^{\prime} \vDash \varphi$,

- $\mathrm{M}, \mathrm{s} \vDash \hat{\mathrm{K}}_{\mathrm{a}} \varphi$ syss existe un $\mathrm{s}^{\prime} \in \mathrm{W}$, tal que $\mathrm{R}_{\mathrm{a}}\left(\mathrm{s}, \mathrm{s}^{\prime}\right) \mathrm{y} \mathrm{M}, \mathrm{s}^{\prime} \vDash \varphi$,

- $\mathrm{M}, \mathrm{s} \vDash[\varphi$ ! $] \mathcal{X}$ syss $\mathrm{M}, \mathrm{s} \vDash \varphi$ implica que $\left.\mathrm{M}\right|_{\varphi}, \mathrm{s} \vDash \mathcal{X}$ 
teniendo en cuenta que $\left.\mathrm{M}\right|_{\varphi}, \mathrm{M}$ restringido a $\varphi$, se define $\left.\mathrm{M}\right|_{\varphi}=\left\langle\mathrm{W}^{\prime}, \Re^{\prime}{ }_{\mathrm{a}}\right.$, $\left.\mathrm{v}^{\prime}\right\rangle$ de manera que:

1. $\mathrm{W}^{\prime}=\{\mathrm{s} \in \mathrm{W}: \mathrm{M}, \mathrm{s} \models \varphi\}$,

2. $\Re^{\prime}{ }_{\mathrm{a}}=\Re_{\mathrm{a}} \cap\left(\mathrm{W}^{\prime} \times \mathrm{W}^{\prime}\right)$, para cada $\mathrm{a} \in \mathrm{G}$,

3. $\mathrm{v}^{\prime}(\mathrm{p})=\mathrm{v}(\mathrm{p}) \cap \mathrm{W}^{\prime}$, para cada $\mathrm{p} \in \mathcal{P}$.

Las relaciones de accesibilidad pueden tener determinadas características (serialidad, reflexividad, transitividad, etc.). Por otra parte, sólo se anuncian públicamente verdades, en ningún caso falsedades. De acuerdo con estas características se identifican clases de marcos de Kripke, para los cuales se establecen ciertas axiomatizaciones. Un sistema axiomático mínimo para lógica epistémica consta de los siguientes (esquemas de) axiomas y reglas (haciendo uso del lenguaje referido):

1. Todas las instancias de las tautologías proposicionales

2. $\mathrm{K}_{\mathrm{a}}(\varphi \rightarrow \psi) \rightarrow\left(\mathrm{K}_{\mathrm{a}} \varphi \rightarrow \mathrm{K}_{\mathrm{a}} \psi\right)$

Además, las reglas

- Regla de modus ponens: de $\varphi \rightarrow \psi$ y $\varphi$, se infiere $\psi$

- Regla de necesitación: de $\varphi$ se infiere $\mathrm{K}_{\mathrm{a}} \varphi$

Si se añaden al sistema mínimo los axiomas que indicamos a continuación, tendremos un sistema epistémico S5 y la clase de marcos de Kripke para la semántica correspondiente, $\mathrm{M}_{\mathrm{S} 5}$ es aquella que contiene los marcos cuyas relaciones de accesibilidad son, además de seriales, reflexivas, transitivas y simétricas. Los nuevos axiomas, relativos al conocimiento, son:

3. $\mathrm{K}_{\mathrm{a}} \varphi \rightarrow \varphi$

4. $\mathrm{K}_{\mathrm{a}} \varphi \rightarrow \mathrm{K}_{\mathrm{a}} \mathrm{K}_{\mathrm{a}} \varphi$

5. $\neg \mathrm{K}_{\mathrm{a}} \varphi \rightarrow \mathrm{K}_{\mathrm{a}} \neg \mathrm{K}_{\mathrm{a}} \varphi$

Estos se corresponden con los habituales T, S4 y S5, respectivamente, de la lógica modal alética. El axioma 4 establece que "si el agente a conoce $\varphi$, entonces este agente conoce que conoce $\varphi$ ", lo que es una expresión de la introspección positiva, mientras que el 5, "si el agente a no conoce $\varphi$, entonces este agente sabe que no conoce $\varphi$ ", se refiere a la introspección negativa. No entramos en la discusión acerca de las razones filosóficas por las cuales se debe aceptar o rechazar la introspección, en particular la negativa; no obstante, esta consideración del conocimiento en contextos inferenciales puede resultar útil, tanto en ámbitos computacionales como epistemológicos. Nótese que estos axiomas determinan condiciones de las relaciones de accesibilidad (como reflexividad, transitividad y simetría). 
El sistema axiomático indicado es ampliable para llegar a constituir un sistema básico de LED. Estos esquemas que se añaden son específicos para anuncios públicos:
6. $[\varphi !] p \leftrightarrow(\varphi \rightarrow p)$
Permanencia atómica
7. $[\varphi !] \neg X \leftrightarrow(\varphi \rightarrow \neg[\varphi !] X)$
Anuncio y $\neg$
8. $[\varphi !](X \wedge \psi) \leftrightarrow([\varphi !] X \wedge[\varphi !] \psi) \quad$ Anuncio $\mathrm{y} \wedge$
9. $[\varphi !] \mathrm{K}_{\mathrm{a}} \chi \leftrightarrow\left(\varphi \rightarrow \mathrm{K}_{\mathrm{a}}[\varphi !] \chi\right) \quad$ Anuncio y conocimiento
10. $[\varphi !][X !] \psi \leftrightarrow[\varphi \wedge[\varphi !] X !] \psi \quad$ Composición de anuncios.

Para determinados propósitos es útil incorporar el tratamiento lógico del conocimiento que se asume por parte de algunos grupos de agentes. Se usan entonces nuevos operadores, el de conocimiento del grupo $\mathrm{E}$, en realidad, la abreviatura de que cada agente del grupo conoce la fórmula de que se trate, el de conocimiento común $\mathrm{C}$, iteración $a d$ infinitum del precedente, y el de conocimiento distribuido $\mathrm{D}$, tal que si, por ejemplo, un agente a conoce $\varphi \rightarrow \psi$ y otro b conoce $\varphi$, se puede decir que el conjunto \{a, b conoce $\psi$. Omitimos los detalles de la semántica de estos operadores en aras de la brevedad.

Se han presentado diversos sistemas de creencias, aunque la idea subyacente es que una proposición creída puede ser verdadera, aunque la proposición como tal sea falsa. Es razonable la afirmación "Juan cree que es martes, aunque de hecho es miércoles", mientras que, tal como hemos usado "conocimiento" más arriba, sería absurdo afirmar "Juan sabe que es martes, aunque de hecho es miércoles" (van Ditmarsch, van der Hoek y Kooi 2008, 38). Para representar las habilidades de los agentes respecto de creencias —aquí haremos uso de ello para el abordaje lógico de la abducción-, se amplía el lenguaje ya conocido con un nuevo operador modal, $\mathrm{B}$, con lo que $\mathrm{B}_{\mathrm{a}} \varphi$ representa que "el agente a cree que $\varphi$ ". Los marcos de Kripke para la interpretación del operador contendrán relaciones $\Re_{\mathrm{Ka}}, \Re_{\mathrm{Ba}} \subseteq \mathrm{W} \times \mathrm{W}$, para el conjunto de mundos $\mathrm{W}$, de manera que las $\Re_{\mathrm{Ka}}$ son las relaciones de accesibilidad respecto del conocimiento, que serán de equivalencia (reflexivas, simétricas y transitivas), mientras que las $\Re_{\mathrm{Ba}}$ son relaciones de accesibilidad respecto de la creencia —en ambos casos, para el agente a-. Por otra parte, para cada agente a, $\Re_{\mathrm{Ba}}$ $\subset \Re_{\mathrm{Ka}}$. Aunque se considere que lo conocido por un agente es creído por él, la afirmación recíproca sería falsa.

El sistema básico KD45 consta de los axiomas antes indicados, aunque cambiando el operador de conocimiento por el de creencia, además de sustituir el axioma 3 por el conocido como axioma D. Es decir, los axiomas son:

1. Todas las instancias de las tautologías proposicionales, 
2. $\mathrm{K}_{\mathrm{a}}(\varphi \rightarrow \psi) \rightarrow\left(\mathrm{K}_{\mathrm{a}} \varphi \rightarrow \mathrm{K}_{\mathrm{a}} \psi\right) \quad$ Axioma $\mathrm{K}$,

3. $\neg \mathrm{B}_{\mathrm{a}} \perp \quad$ Axioma $\mathrm{D} \longrightarrow$ consistencia de creencias-

4. $\mathrm{B}_{\mathrm{a}} \varphi \rightarrow \mathrm{B}_{\mathrm{a}} \mathrm{B}_{\mathrm{a}} \varphi$ introspección positiva,

5. $\neg \mathrm{B}_{\mathrm{a}} \varphi \rightarrow \mathrm{B}_{\mathrm{a}} \neg \mathrm{B}_{\mathrm{a}} \varphi \quad$ introspección negativa.

Aunque se mantienen las reglas de modus ponens y necesitación, esta última es relativa al operador de creencia, de $\varphi$ se infiere $\mathrm{B}_{\mathrm{a}} \varphi$.

De cara a las aplicaciones epistemológicas —más abajo añadimos algunas observaciones sobre este punto-, mediante LED podemos abordar los problemas de los que ha ocupado el modelo AGM de revisión de creencias (elaborado en Alchourrón, Gärdenfors y Makinson 1985; véase también Schurz 2011), que contempla tres operaciones epistémicas, a saber, expansión, contracción y revisión. Se parte de un conjunto $K$ de fórmulas del correspondiente lenguaje, una base de conocimiento, el cual es cerrado bajo consecuencia lógica (en sentido clásico), es decir, $K=\mathrm{Cn}(K)$. En esta breve presentación seguimos fundamentalmente el resumen expuesto en van Ditmarsch y Nepomuceno (2019). La expansión de $K$ con una fórmula $\varphi$, en símbolos $K+\varphi$ es el conjunto más pequeño de fórmulas que verifica:

1. $K+\varphi$ es una base de conocimiento (cerrado bajo consecuencia).

2. $\varphi \in K+\varphi$.

3. $K \subseteq K+\varphi$.

4. Si $\varphi \in K$, entonces $K+\varphi=K$.

5. Si $K^{\prime} \subseteq K$, entonces $K^{\prime}+\varphi \subseteq K+\varphi$.

Por otra parte, la contracción de $K$ con la fórmula $\varphi$, en símbolos $K-\varphi$, da lugar a un conjunto de fórmulas que ha de verificar los siguientes postulados - solamente prestamos atención a los que han sido ampliamente aceptados, pues algunos de los iniciales opuestos en el modelo AGM han sido muy discutidos-,

1. $K-\varphi$ es una base de conocimiento.

2. $K-\varphi \subseteq K$.

3. Si $\varphi \notin K$ (o bien $K \nvdash \varphi)$, entonces $K-\varphi=K$.

4. Si $\varphi \in K$, entonces $K \subseteq(K-\varphi)+\varphi$.

5. $\mathrm{Si} \models \varphi \leftrightarrow \psi$, entonces $K-\varphi=K-\psi$. 
La revisión de una base de conocimiento $K$ mediante la fórmula $\varphi$, en símbolos $K * \varphi$ se define mediante la identificación de Levi, como una contracción seguida de una expansión; en concreto,

$$
K * \varphi=(K-\neg \varphi)+\varphi .
$$

\section{Sobre lógica abductiva}

Se han presentado diversas formas de tratamiento lógico de la abducción, aunque se pueden clasificar en dos grupos: el clásico, denominado modelo AKM - de Aliseda, Kakas-Kowalski y Meheus-Maganani- y el conocido esquema GW - Gabbay y Woods-. En Aliseda (2006) y en Soler (2012) se define la abducción como se concibe en el modelo AKM, se trata de un proceso que produce explicaciones abductivas específicas con cierta estructura inferencial. Un problema abductivo, a su vez "desencadenante" de la solución abductiva, se puede representar como la terna $\left(\Theta, \varphi, \vdash_{\mathrm{X}}\right)$, donde $\Theta$ representa una teoría base o base de conocimiento, un conjunto de fórmulas de un lenguaje dado, $\varphi$ representa el fenómeno a explicar, un hecho "sorprendente" (como originalmente lo denominó Peirce) en el sentido de que no queda explicado (inferencialmente) por la teoría, mientras que $\vdash_{X}$ se refiere a la lógica subyacente de la práctica científica de que se trate, es decir, el sistema lógico que rige la comunidad científica en cuyo seno se realiza la práctica en cuestión (Hernández y Nepomuceno 2011). Una vez que se obtiene una solución $\psi$, de la teoría y la solución se puede inferir — según la lógica subyacente- $\varphi$. Es decir, se verificará

$$
\text { si } \Theta, \varphi \Rightarrow_{\text {abd }} \psi \text {, entonces } \Theta, \psi \vdash_{\mathrm{X}} \varphi \text {, }
$$

donde $\Rightarrow_{\text {abd }}$ expresa la inferencia abductiva propiamente dicha. Ahora bien, para que la abducción sea explicativa se debe cumplir que $\Theta \nvdash_{x} \varphi$ y que $\psi \nvdash_{x} \varphi$, de esta manera se evitan las explicaciones triviales. Una propuesta de tratamiento de la abducción en este marco mediante GTS lo hallamos en Hernández y Nepomuceno (2011).

En el planteamiento del esquema GW - sucintamente presentado en Barés-Gómez y Fontaine (2017) — , la abducción es una forma de inferencia basada en agentes y orientada hacia ciertos objetivos y no tiene por qué concluir en una proposición o en nuevo conocimiento, incluso podría no ser explicativa. Se alcanza una hipótesis que se toma como base para nuevas acciones, a pesar de que pueda persistir la ignorancia oficial; es una hipótesis que se puede utilizar y divulgar en otros razonamientos y acciones. El agente adopta la hipótesis obtenida mediante un razonamiento abductivo y queda momentáneamente como base para nuevas actuaciones cognitivas. Precisamente en Barés Gómez y Fontaine (2017) hallamos una aplicación de lógica dialógica para dar cuenta de desarrollos de la abducción tipo GW. 
Se pueden considerar como complementarios ambos planteamientos. En cualquier caso, una certera caracterización de la abducción viene dada por las tesis de Kapitan-Hintikka (Hintikka 1998), que indicamos a continuación:

- Tesis inferencial. La abducción es, o incluye, un proceso inferencial, o varios procesos inferenciales.

- Tesis de propósito. En la investigación científica, el propósito de la abducción es doble: (i) generar nuevas hipótesis, y (ii) seleccionar hipótesis para su posterior verificación.

- Tesis de comprehensión. La abducción científica incluye todas las operaciones mediante las cuales se generan las teorías.

- Tesis de autonomía. La abducción es, o incorpora, un tipo de razonamiento que es distinto, e irreductible, a la inducción y a la deducción.

El fenómeno a explicar (el hecho sorprendente), desencadenante del proceso abductivo, si se presenta como una proposición (una fórmula del lenguaje de que se trate) y la hipótesis o conjetura alcanzada mediante abducción constituye el principal elemento explicativo, puede ser de dos clases, a saber, novedad o anomalía (Aliseda 2006). Simbólicamente, para el problema abductivo $\left(\Theta, \varphi, \vdash_{\mathrm{X}}\right)$, tendremos:

1. Una novedad, si $\Theta \nvdash_{\mathrm{x}} \varphi \mathrm{y} \Theta \nvdash_{\mathrm{x}} \neg \varphi$.

2. Una anomalía, si $\Theta \nvdash_{x} \varphi$ pero $\Theta \vdash_{x} \neg \varphi$.

A partir de esta distinción es fácilmente equiparable la tarea de la búsqueda de soluciones a problemas abductivos, en el marco del modelo AKM de abducción, con un proceso de revisión de creencias, si bien considerando algunas restricciones para dar lugar a las nociones de AGM relativas a la abducción (Aliseda 2006). A este respecto, dado un problema abductivo $\left(\Theta, \varphi, \vdash_{\mathrm{X}}\right)$, una solución abductiva $\psi$ permite la explicación de $\varphi$, en el caso de ser una novedad, mediante una expansión abductiva, para abreviar, ExpAb $(\Theta, \varphi$, $\vdash_{\mathrm{X}}$ ), como

$$
\operatorname{ExpAb}\left(\Theta, \varphi, \vdash_{\mathrm{X}}\right)=\left\{\psi \in \mathrm{L}(\Theta): \Theta, \psi \vdash_{\mathrm{X}} \varphi\right\},
$$

donde $L(\Theta)$ representa el lenguaje de la teoría base $\Theta$ y se cumplen las restricciones de la abducción explicativa. En el caso de la contracción, cuando $\varphi$ representa una anomalía $-\Theta \vdash_{\mathrm{X}} \neg \varphi-$, sea el conjunto $\left\{\beta_{1}, \ldots, \beta_{\mathrm{n}}\right\} \subset \Theta$ tal que $\Theta \backslash\left\{\beta_{1}, \ldots, \beta_{\mathrm{n}}\right\} \forall_{\mathrm{X}} \neg \varphi$. De este modo,

$$
\operatorname{ContrAb}_{-\varphi}=\Theta \backslash\left\{\beta_{1}, \ldots, \beta_{\mathrm{n}}\right\} .
$$

Puesto que ante una anomalía se necesita una revisión, adaptamos la identidad de Levi, de manera que

$$
\operatorname{RevAb}\left(\Theta, \varphi, \vdash_{\mathrm{X}}\right)=\operatorname{ExpAb}\left(\left(\operatorname{ContrAb}_{-\varphi}\right), \varphi, \vdash_{\mathrm{X}}\right) .
$$


Desde el punto de vista de la LED es factible redefinir el lenguaje con las operaciones epistémicas de expansión, contracción y revisión, las cuales se representan para una fórmula $\varphi$, respectivamente, por $+\varphi,-\varphi$ y $* \varphi$, por lo que las expresiones $[+\varphi] \psi,[-\varphi] \psi \mathrm{y}[* \varphi]$ $\psi$ deben leerse como "tras cada expansión con $\varphi, \psi$ es el caso", "tras cada contracción con $\varphi, \psi$ es el caso" y "tras cada revisión con $\varphi, \psi$ es el caso", respectivamente. Teniendo en cuenta una vez más la identidad de Levi, cabe entender $[* \varphi] \psi=[-\neg \varphi][+\varphi] \psi$. Los correspondientes operadores duales, $\langle+\varphi\rangle,\langle-\varphi\rangle \mathrm{y}\langle * \varphi\rangle$, se definen como es habitual.

En cuanto a la semántica de los nuevos operadores, se han de tener en cuenta las acciones mismas. Para un modelo $\mathrm{M}=\left(\mathrm{W}, \Re_{\mathrm{a}}, \mathrm{v}\right)$ y estado $\mathrm{s}$,

$$
\mathrm{M}, \mathrm{s} \vDash[+\varphi] \psi \text { syss si } \mathrm{M}, \mathrm{s} \models \varphi \text {, entonces }\left.\mathrm{M}\right|_{+\varphi}, \mathrm{s} \models \psi,
$$

para $\left.\mathrm{M}\right|_{+\varphi}=\left(\mathrm{W}^{+\varphi}, \Re^{+\varphi}, \mathrm{v}^{+\varphi}\right), \mathrm{W}^{+\varphi}=\mathrm{W} \cup \mathrm{W}^{\prime}$, tal que para todo $\mathrm{s}^{\prime} \in \mathrm{W}^{\prime}, \mathrm{s}^{\prime} \in \mathrm{v}^{+\varphi}(\varphi) \mathrm{y}$, para toda variable proposicional $\mathrm{p}, \mathrm{v}^{+\varphi}(\mathrm{p})=\mathrm{v}(\mathrm{p})$ si $\mathrm{p}$ no ocurre en $\varphi\left(\right.$ en otro caso $\mathrm{v}^{+\varphi}(\mathrm{p})=$ $\left.v(p) \cup W^{\prime}\right)$. Es decir, se trata de acciones epistémicas que permiten una actualización de la información en las que determinadas fórmulas valen. De manera análoga se ha de considerar la contracción:

$$
\mathrm{M}, \mathrm{s} \models[-\varphi] \psi \text { syss } \mathrm{M}, \mathrm{s} \models \varphi, \text { entonces }\left.\mathrm{M}\right|_{-\varphi}, \mathrm{s} \models \psi .
$$

Ahora $\mathrm{W}^{-\varphi}=\mathrm{W} \backslash\left\{\beta_{1}, \ldots, \beta_{\mathrm{n}}\right\}$, siempre que $\mathrm{W} \backslash\left\{\beta_{1}, \ldots, \beta_{\mathrm{n}}\right\}$ sea el mínimo conjunto tal que $\mathrm{W} \backslash\{\beta 1, \ldots, \beta \mathrm{n}\} \not \models \varphi$. Por lo que respecta a la revisión, tendremos

$$
\mathrm{M}, \mathrm{s} \vDash[* \varphi] \psi \text { syss } \mathrm{M}, \mathrm{s} \models[-\neg \varphi][+\varphi] \psi .
$$

Con estos nuevos operadores las nociones de expansión, contracción y revisión abductivas se pueden presentar en el siguiente formato. Sea $M_{\Theta}$ un modelo de la teoría $\Theta$. Entonces, para el problema abductivo correspondiente,

- $\operatorname{ExpAb}\left(\Theta, \varphi, \vdash_{\mathrm{X}}\right)=\left\{\psi \in \mathrm{L}(\Theta): \mathrm{M}_{\Theta}, \mathrm{s} \vDash[+\psi] \varphi\right\}$, si $\varphi$ es una novedad

- $\operatorname{RevAb}\left(\Theta, \varphi, \vdash_{\mathrm{X}}\right)=\left\{\psi \in \mathrm{L}(\Theta): \mathrm{M}_{\Theta}, \mathrm{s} \models[-\neg \varphi][+\psi] \varphi\right\}$, en el caso de que $\varphi$ sea una anomalía.

\section{Una anomalía: la lengua pirahã}

En este apartado vamos a aplicar el aparato teórico expuesto anteriormente a un debate abierto en el ámbito de la lingüística, con motivo de presentar un proceso de razonamiento abductivo en una investigación científica concreta. Antes de comenzar, vamos a asumir, como presupuesto básico, que el paradigma actual de la gramática generativa es la teoría de Chomsky $\mathrm{T}(\mathrm{Ch})$.

La teoría de la gramática generativa defiende que existe una estructura del lenguaje universal, que nos permite, desde que somos pequeños, adquirir el lenguaje. En este senti- 
do, la teoría defiende que todos los seres humanos tenemos la facultad innata del lenguaje, con lo que el lenguaje no sería una construcción humana desde cero, un artefacto humano, sino el desarrollo de un hipotético "órgano del lenguaje", que estaría ubicado en alguna parte del cerebro. Para reforzar nuestra propuesta de estudiar un problema lingüístico como un problema abductivo, vamos a señalar lo siguiente: el propio Chomsky, en su defensa de un lenguaje universal innato, aludió a Peirce a la hora de defender que aprendemos el lenguaje gracias a la capacidad humana de elegir las mejores hipótesis, de entre las infinitas hipótesis posibles, acerca de un tema: "Abduction also finds a place in theories of language acquisition. Most prominently, Chomsky proposed that learning a language is a process of theory construction. A child 'abduces' the rules of grammar guided by her innate knowledge of language universals" (Aliseda 2006, 44).

De todo ello, por tanto, se deriva según Chomsky la existencia de una estructura gramática común a todas las lenguas, por lo que se podrían definir leyes fundamentales de la gramática que estuvieran presentes en cualquiera. Una de las leyes o principios que Chomsky estableció como universal a toda lengua fue el principio de la recursividad, que consiste en la capacidad de poner una frase dentro de otra en una cadena infinita, a partir de las herramientas finitas del lenguaje.

No obstante, el lingüista y antropólogo, Daniel L. Everett, presentó, como crítica a la teoría chomskiana, el caso de la lengua de una tribu amazónica: la lengua pirahã. El estudio de Everett de la lengua pirahã establece que la gramática de esta se rige por un principio propio de la cultura pirahã, a saber, el principio de inmediatez de la experiencia. Según este principio un miembro de la tribu solo habla acerca de aquello de lo que tiene una experiencia directa o de testimonios comunicados por alguien que puede corroborar lo que el otro dice: "Los enunciados pirahã contienen únicamente afirmaciones directamente relacionadas con el momento en que se habla, tanto si se trata de una experiencia personal del hablante como de un hecho presenciado por un contemporáneo del hablante" (Everett 2014, 165). Este principio rector de las comunicaciones se ve reflejado en la gramática, según Everett, mediante la ausencia de cláusulas recursivas y oraciones subordinadas. Además, los conceptos de los pirahã se relacionan con la experiencia inmediata, con lo que no aceptan ningún nivel de abstracción más allá del que supone la creación misma de los conceptos que poseen.

En último término, se podría decir que el lenguaje pirahã respondería perfectamente a la proposición de Wittgenstein, según la cual los límites de nuestro mundo son los límites de nuestro lenguaje. El lenguaje pirahã, como afirma Everett, no representa nada que no sea accesible a la experiencia y, con ello, tampoco representa nada que no sea accesible a la cultura pirahã. En definitiva, la cultura pirahã, en concreto el principio de inmediatez de la experiencia, determinaría el lenguaje pirahã, de tal forma que, según Everett, la teoría chomskiana de la gramática universal, sustentada en el principio de la recursividad, quedaría en entredicho. En palabras de Everett: 
La lengua es el resultado de las sinergias entre los valores de una sociedad, la teoría de la comunicación, la biología, la fisiología, la física (las limitaciones propias de nuestro cerebro y nuestra capacidad fonética) y el pensamiento humano. Y creo que esto también es válido para el motor del lenguaje: la gramática. (Everett 2014, 253)

Aplicando los instrumentos teóricos que hemos expuesto, nos encontramos ante un problema abductivo $\left(\mathrm{T}(\mathrm{Ch}), \varphi_{\mathrm{p}}, \vdash_{\mathrm{TL}}\right)$, en el que, dada la teoría de Chomsky, $\mathrm{T}(\mathrm{Ch})$, la lógica subyacente de la teoría lingüística, $\vdash_{\mathrm{TL}}$, el caso de la lengua pirahã constituye un fenómeno discrepante, $\varphi_{\mathrm{p}}$, con respecto a la teoría, debido a la ausencia de cláusulas recursivas y al condicionamiento fuerte de la gramática pirahã por la cultura. En definitiva, tenemos que $\mathrm{T}(\mathrm{Ch}) \forall_{\mathrm{TL}} \varphi_{\mathrm{p}}$ y nuestro propósito a este respecto es encontrar una solución abductiva, $\psi$, al problema.

Después de la aparición del trabajo de Everett, se produjo dentro de la esfera de Chomsky un proceso que podríamos denominar de revisión parcial de creencias, ya que se produjo una adaptación o concreción de la teoría chomskiana para dar cuenta del fenómeno de la gramática pirahã. Dentro de este proceso, el artículo más destacado fue el de los lingüistas Andrew Nevins, David Pesetsky y Cilene Rodrigues (NPR), los cuales cuestionaron desde el primer momento la identificación del lenguaje pirahã como un fenómeno sorprendente o anómalo. El grupo NPR comienza su respuesta a Everett afirmando que dentro de la teoría de la gramática universal se asume el hecho de que la gramática se forma o moldea en parte por la experiencia (véase Nevins, Pesetsky y Rodrigues 2009, $359)$.

En primer lugar, el grupo NPR afirma que las características del pirahã, que para Everett eran excepcionales, son fenómenos ya identificados en otras lenguas, con lo que serían problemas ya resueltos por la teoría $\mathrm{T}(\mathrm{Ch})$ o en proyecto de ser resueltos. Una de las cuestiones que el grupo NPR considera ya examinada en otras lenguas, y estudiada dentro de la teoría, es la cuestión referida a la conexión entre cultura y gramática, que para Everett es central en su teoría. La postura que el grupo NPR defiende es que la teoría de Chomsky, T(Ch), es una teoría más abierta y amplia de lo que Everett considera. En relación con lo que acabamos de exponer, el grupo NPR alude a la regla estructural en la construcción de frases, introducida por Chomsky en 2002, conocida como la regla Merge. De este modo, la recursividad queda asociada a una regla estructural, cuya aplicación no tiene por qué ser necesaria, en vez de ser definida como un principio fundamental de la teoría de la gramática generativa:

Merge takes two linguistic units as input and combines them to form a set (a phrase), in which one element is designated as the phrase's head. Two kinds of linguistic units may serve as input to Merge: (i) lexical items, and (ii) phrases formed by previous applications of Merge. Since Merge may take previous applications of Merge as input, the rule is recursive. Iterated Merge yields the full variety of phrase 
structures studied in syntactic research - structures composed of lexical items and phrases that were themselves produced by Merge. (Nevins, Pesetsky y Rodrigues 2009, 365)

Con todo ello el grupo NPR quiere dejar claro que la naturaleza de la gramática universal está sujeta a nuevas interpretaciones, y que hay debates abiertos acerca de su capacidad de explicar cada uno de los fenómenos lingüísticos. Como señala el grupo NPR, no hay un modelo general de la gramática universal (véase Nevins, Pesetsky y Rodrigues $2009,357)$, por lo que no estamos ante un sistema de conocimientos organizado deductivamente, al modo de la geometría de Euclides, por ejemplo:

As a logical matter, of course, it is possible that beliefs considered nonnegotiable will turn out to be false, and it is never good to be so rigid about one's expectations that it becomes impossible for a new discovery to offer the element of surprise. One might disagree with Everett's claims about universal grammar, reject the IEP [immediacy of experience principle] and the claimed link between culture and grammar, and still agree that Pirahã grammar presents us with just such a surprise (in which case, one might wish to rethink aspects of syntactic theory in light of the new results). (Nevins, Pesetsky y Rodrigues 2009, 359)

En este sentido, el grupo NPR utiliza una lógica subyacente, $\vdash_{\mathrm{TL}}$, que responde a los criterios de una lógica abductiva. Además, el grupo NPR no niega la posibilidad de añadir nuevas hipótesis explicativas a la teoría de la gramática universal para explicar nuevos fenómenos lingüísticos. Sin embargo, lo que niega el grupo de lingüistas es que el lenguaje pirahã constituya un fenómeno sorprendente, $\varphi_{\mathrm{p}}$, que requiera una explicación particular; más bien debería hablarse de la necesidad de aplicar de una manera diferente la regla Merge. En este sentido, dada la teoría de Chomsky de la gramática generativa, con la regla estructural Merge, $\mathrm{T}(\mathrm{Ch})_{\mathrm{M}}$, y la hipótesis asumida dentro de la teoría, según la cual la experiencia, así como la cultura, interviene en la configuración de la gramática, $\psi$, tenemos que la existencia del lenguaje pirahã, $\varphi_{\mathrm{p}}$, es un fenómeno que, según NPR, entronca con lo anterior

$$
\mathrm{T}(\mathrm{Ch})_{\mathrm{M}}, \psi \vdash_{\mathrm{TL}} \varphi_{\mathrm{p}} .
$$

Por tanto, el fenómeno pirahã constituiría una novedad asumida por la teoría, teniendo en cuenta la hipótesis $\psi$ que Everett no consideraba, es decir, $\mathrm{T}(\mathrm{Ch})_{\mathrm{M}} \nvdash_{\mathrm{TL}} \varphi_{\mathrm{p}} \mathrm{y} \mathrm{T}(\mathrm{Ch})$ ${ }_{\mathrm{M}} \forall_{\mathrm{TL}} \neg \varphi_{\mathrm{p}}$, que solo requeriría una nueva configuración de las reglas estructurales de la gramática universal.

No obstante, el hecho de que la aparición de los estudios de Everett acerca del pirahã, siga siendo fuente de debates y discusiones dentro de la lingüística nos lleva a la siguiente pregunta: ¿es la lengua pirahã efectivamente una novedad o es más bien una anomalía? En este trabajo defendemos que el caso de la lengua pirahã es una anomalía vista o estudiada desde la propia teoría paradigmática, hablando en términos kuhnianos, como un reto o 
rompecabezas a partir del cual se pueden seguir dos caminos: llevar a cabo un proceso de expansión abductiva de la teoría - como el grupo NPR - o llevar a cabo un proceso de revisión abductiva de la teoría chomskiana, que sería un camino más afín al proyecto de Everett. Por tanto, podríamos concluir que, dado que existe todavía un debate abierto, generado por el estudio de Everett, acerca de la capacidad de la teoría chomskiana de explicar fenómenos lingüísticos que no se infieren directamente de la teoría, como es el caso del pirahã, no podemos más que seguir considerando el pirahã como una anomalía, que no invalida la teoría chomskiana, pero que tampoco consigue hacer que lo anómalo se vuelva esperado:

$$
\mathrm{T}(\mathrm{Ch})_{\mathrm{M}} \nvdash_{\mathrm{TL}} \varphi_{\mathrm{p}} \text { pero } \mathrm{T}(\mathrm{Ch})_{\mathrm{M}} \vdash_{\mathrm{TL}} \neg \varphi_{\mathrm{p}} .
$$

\section{Conclusiones y trabajo futuro}

Tras el giro dinámico en la lógica contemporánea vemos que en la investigación científica no podemos considerar como relevante la lógica clásica en exclusiva. La riqueza conceptual que se alcanza con el desarrollo de las lógicas no clásicas constituye un acicate para nuevas perspectivas en estudios epistemológicos. Como columnas en las que se sustenta plenamente la consumación del giro dinámico, la IF-logic (y semántica GTS), la lógica dialógica y el programa de dinámica lógica de la información, con los desarrollos de la LED como su expresión fundamental, ya han sido usadas en el estudio de la abducción, respectivamente en Barés Gómez y Fontaine (2017), Hernández y Nepomuceno (2011), y en Nepomuceno, Soler y Velázquez (2017) —en este mismo trabajo hemos explorado más de una línea de abordaje de la abducción con herramientas de LED-.

El sistema básico de LED que hemos presentado nos puede servir como una herramienta inicial para la consideración de una lógica subyacente en ciertas prácticas científicas. Por práctica científica hemos de entender la ejecución de un programa de investigación que realiza una comunidad científica, la cual se puede representar como un conjunto de agentes, en terminología de los sistemas multiagente. Por lógica subyacente debemos entender el conjunto de normas que rigen los modos de inferir de la comunidad en la práctica de que se trate. De esta manera, en cierto sentido, el sistema inferencial de la comunidad científica que lleva a cabo la práctica en cuestión es representable como una lógica. La continuidad de estas aproximaciones y aplicaciones deberá dirigir nuevas exploraciones, por ejemplo, con anuncios, y otras acciones epistémicas, realizadas por agentes concretos para grupos (no necesariamente el anuncio público como tal, sino dirigido a una clase de agentes). Asimismo, la consideración del tiempo, es decir, el uso de sistemas de lógica epistémica temporal.

Tanto los sistemas multiagente como, por ejemplo, el modelo AGM de revisión de creencias o sus variantes (véase, por ejemplo, Luna 2001), para estudios epistemológicos, se toman en préstamo de temáticas que fueron en su momento estudiadas en el ámbito de la Inteligencia Artificial (IA). Ello se justica por la representatividad que cabe atribuir a la 
IA respecto de la historia de la ciencia en el pensamiento occidental. $\mathrm{Si}$, como creemos, en la historia de la IA se condensan las principales problemáticas surgidas en la historia de la ciencia occidental, entonces cabe considerar la primera como un marco de referencia de estudios epistemológicos. Se trata de una cuestión de economía y eficiencia, ya que la IA tiene una fecha de nacimiento, 1956, con motivo de la famosa Conferencia de la Universidad de Dartmouth, y sesenta y tres años son más fáciles de abarcar que más de veinte siglos. En trabajos futuros se pueden explorar casos concretos de la historia de la IA calificables como de revisión de creencias. A este respecto, los sistemas de lógica defectuosa y su uso en el estudio de la abducción constituyen una línea abierta de indudable interés epistemológico.

En algunos de los usos de los sistemas estudiados se nos plantea un importante problema, el de la omnisciencia lógica. En el modelo AGM el agente haría las operaciones epistémicas a partir de bases de conocimiento cerradas bajo consecuencia, es decir, se trataría de un agente que es lógicamente omnisciente. Pero los agentes reales, los miembros de la comunidad científica, no lo son. En este caso, la propia LED nos permite extraer más herramientas conceptuales. Si un agente conoce una regla de inferencia R que, en general, podemos enunciar como "de precondición-R se infiere postcondición-R", entonces cabe pensar que el agente (no omnisciente) alcanza la postcondición-R siempre que, a partir de precondición- $\mathrm{R}$, realice determinada acción. Sea ?R la acción de comprobar y asumir la regla de inferencia indicada, es decir, aprenderla; entonces se añadirán expresiones para acciones $\alpha$-la más básica, aprender una regla, concatenación de acciones, etc.—, de manera que $[\alpha] \varphi$ expresará que "tras la acción $\alpha, \varphi$ es el caso" (una presentación concreta de un sistema de lógica de acciones aparece en van Ditmarsch, van der Hoek y Kooi 2008, 190 y ss.). A partir de aquí otra línea de trabajo debería conectar toda esta temática de los modelos de acción con el tratamiento lógico de la abducción. En conexión con ello, investigar las posibilidades de desarrollo de diálogos lógicos de carácter colaborativo y revisable (con posibles aplicaciones en tecnologías del lenguaje).

El sistema con operadores de expansión, contracción y revisión no calca plenamente, por así decir, las características de las correspondientes operaciones del modelo AGM. No obstante, en este contexto, precisamente el problema de la omnisciencia lógica recomienda un tratamiento diferente del original. Lo que nos interesan como punto de partida son las teorías base equiparables con teorías científicas, no ya cerradas bajo consecuencia, sino simplemente consistentes (lo que no excluye que en algunos contextos interesen sistemas paraconsistentes, lo que hemos excluido en este trabajo). Si bien las operaciones de expansión, contracción y revisión en AGM se rigen por unos postulados específicos sobre conjuntos de fórmulas, los operadores $+\varphi$ y $-\varphi$ y, por ende, $* \varphi$, que hemos incorporado en LED actúan sobre estructuras kripkeanas. Como quiera que no parece posible hallar una solución inequívoca al problema de la omnisciencia lógica, tal vez lo más razonable sea abordar las situaciones prácticas mediante la elección de los marcos y modelos disponibles que más o menos capten el grado de no-omnisciencia lógica aceptable en esa situación particular (Meyer, van der Hoek 2004, 89). Así, en la búsqueda de soluciones a 
problemas abductivos (en el marco AKM) contamos con los sistemas comentados (LED con los diez esquemas axiomáticos, KD45, ampliación con acciones epistémicas, etc.). A este respecto, el sistema KL (Kraus y Lehmann, estudiado en Meyer, van der Hoek 2004) ofrece un intento de combinar conocimiento y creencia de una manera lo más realista posible y señala un nuevo sendero de trabajos futuros.

Hemos presentado de manera resumida el caso de la lengua pirahã, en el que se suscitan ciertos problemas epistemológicos de los que nos ocupamos en el trabajo. Su aparición como hecho sorprendente nos lleva a preguntarnos si hemos de considerarlo como una novedad y, en consecuencia, debemos buscar la solución como una expansión abductiva, o si se trata más bien de una anomalía, en cuyo caso se ha de proceder a una revisión de creencias. Tanto si se aborda el problema desde una perspectiva chomskiana o su opuesta, más afín a las conclusiones de Everett, entendemos que el enfoque correcto es el del tratamiento del hecho como una anomalía. A partir de aquí, cabe una más amplia y detallada explicación de este fenómeno en la que entrarán en juego buena parte de las expectativas antes referidas.

\section{Referencias bibliográficas}

Aho, T., Pietarinen, A. (eds.) (2006). Truth and Games. Essays in Honor of Gabriel San$d u$. Acta Philosophica Fennica, Vol. 78. Helsinki: Societas Philosophica Fennica.

Alchourrón, C. E., Gärdenfors, P., Makinson, D. (1985). On the logic of theory change: partial meet contraction and revision functions. Journal of Symbolic Logic, 50(2): 510-530. doi: $10.2307 / 2274239$

Aliseda, A. (2006). Abductive Reasoning. Logical Investigations into Discovery and Explanation. Synthese Library, Vol. 330. Dordrecht: Springer.

Aliseda, A. (2014). La Lógica como Herramienta de la Razón. Razonamiento Ampliativo en la Creatividad, la Cognición y la Inferencia. Cuadernos de lógica, epistemología y lenguaje, Vol. 6. Londres: College Publications.

Baltag, A., Smets, S. (2012). The dynamic turn in quantum logic. Synthese, 186: 753-773. doi: $10.1007 / \mathrm{s} 11229-011-9915-7$

Barés Gómez, C., Fontaine, M. (2017). Argumentation and Abduction in Dialogical Logic. In L. Magnani, T. Bertolotti (eds.), Springer Handbook of Model-Based Science, 295-314. Dordrecht: Springer.

van Benthem, J. (2011). Logical Dynamics of Information and Interaction. Cambridge: Cambridge University Press.

van Ditmarsch, H., van der Hoek, W., Kooi, B. (2008). Dynamic Epistemic Logic. Synthese Library, Vol. 337. Dordrecht: Springer. doi: 10.1007/978-1-4020-5839-4 
van Ditmarsch, H., Nepomuceno, A. (2019). Public announcements, belief expansion and abduction. En O. Pombo, A. Pato y J. Redmond (eds.), Epistemologia, Lógica e Linguagem, pp. 151-161. Lisboa: Centro de Filosofia das Ciências da Universidade de Lisboa.

Everett, D. L. (2014). 'No duermas, hay serpientes' Vida y lenguaje en la Amazonia. Madrid: Turner Publicaciones S.L.

Fagin, R., Halpern, J. Y., Moses, Y., Vardi, M. Y. (1995). Reasoning about Knowledge. Cambridge, London: The M. I. T. Press.

Gochet, P. (2002). The dynamic turn in century logic. Synthese, 130: 175-184. doi: 10.1023/A:1014435213120

Hernández, I., Nepomuceno, A. (2011). Lógicas alternativas subyacentes a las prácticas científicas. En O. Pombo (ed.), Lógica Universal e Unidade da Ciencia, pp. 121140. Lisboa: Centro de Filosofia das Ciências da Universidade de Lisboa.

Hintikka, J. (1973). Logic, Language-Games and Information. Oxford: Clarendon Press.

Hintikka, J. (1997). A revolution in the foundation of mathematics? Synthese, 111: 155170. doi: 10.1023/A:1004970403579

Hintikka, J., Sandu, G. (1997). Game-Theoretical Semantics. In J. van Benthem and A. ter Meulen (eds.), Handbook of Logic and Language, pp. 361-410. Amsterdam: Elsevier.

Hintikka, J. (1998). What is abduction? The fundamental problem of contemporary epistemology. Transactions of The Charles S. Peirce Society, 34(3): 503-533. doi: 10.1007/978-94-015-9313-7_4

Luna, C. D. (2001). Una Generalización del Modelo AGM de Cambio de Creencias. Inteligencia Artificial: Revista Iberoamericana de Inteligencia Artificial, 5(13): 23-32.

Marion, M. (2006). Hintikka on Wittgenstein: From Language-Game to Game Semantics. Acta Philosophica Fennica, 78: 255-274.

Meyer, J. J. Ch., van der Hoek, W. (2004). Epistemic Logic for AI and Computer Science. Cambridge: Cambridge University Press.

Nepomuceno, A., Soler, F., Velázquez, F. R. (2017). Abductive Reasoning in Dynamic Epistemic Logic. In L. Magnani and T. Bertolotti (eds.), Springer Handbook of Model-Based Science, pp. 269-293. Dordrecht: Springer, doi: 10.1007/978-3-31930526-4-13

Nevins, A., Pesetsky, D., Rodrigues, C. (2009). Pirahã exceptionality: A reassessment. Language, 2(85): 355-404.

Popper, K. R. (1980). La lógica de la investigación científica. Madrid: Tecnos. 
Rahman, S., Clerbout, N., Keiff, L. (2009). Dialogues and Natural Deduction. In G. Primiero (ed.), Acts of Knowledge, History, Philosophy, Logic, pp. 301-336. London: College Publication.

Redmond, J. and M. Fontaine (2011). How to Play Dialogues. An Introduction to Dialogical Logic. Londres: College Publication.

Saarinen, E. (ed.) (1979). Game-Theoretical Semantics. Essays on Semantics by Hintikka, Carlson, Peacocke, Rantala, and Saarinen. Dordrecht: Springer.

Schurz, G. (2011). Abductive Belief Revision in Science. In E. J. Olsson and S. Enqvist (eds.), Belief Revision meets Philosophy of Science, pp. 77-104. Dordrecht: Springer. doi: 10.1007/978-90-481-9609-8-4

Soler, F. (2012). Razonamiento abductivo en lógica clásica. Londres: College Publications. 